\title{
Antimicrobial Resistance Pattern of Shigella species Over Five Years at a Tertiary-care Teaching Hospital in North India
}

Sir,

Shigellosis is a global human health problem, especially in developing countries where there are inadequate hygiene and unsafe water supplies $(1,2)$. Shigella accounts for a significant proportion of cases of bacillary dysentery in many tropical and subtropical countries $(3,4)$. Shigella is one of the most important causes of gastroenteritis-induced deaths in 3-5 million children aged less than five years in developing countries (5). The emergence of multiple drug resistance to cost-effective antimicrobials against Shigella is a matter of concern in developing countries (6). It is also well-documented that pandemic strains often exhibit multiple antimicrobial resistance and induce severe illness with high case fatality in all age-groups (7). In the tropics, most infections occur due to $S$. flexneri and infections that occur primarily due to Shigella sonnei are less common $(8,9)$.

Over a decade ago, results of active surveillance studies showed that, in most endemic countries, especially in Asia and sub-Saharan Africa, there was an emergence of multidrug resistance to different antimicrobials, including ampicillin, co-trimoxazole, and nalidixic acid (9).

Besides the temporal changes in the antibiogram of Shigella species, it is well-known that antimicrobial susceptibility patterns in Shigella may differ between geographical areas. Such differences are never stable and may change rapidly, especially in places where antimicrobials are used excessively, particularly in developing countries (1). This warrants for robust monitoring of the pattern of antibiogram for this organism.

Hence, the present study was conducted to determine the incidence and prevalence of Shigella serogroups isolated from cases of dysentery during

Correspondence and reprint requests should be addressed to:

Dr. Sonal Saxena

Flat 6, Deluxe Apartments

Vasundhara Enclave

Delhi 110096

India

Email: sonalsaxena3@rediffmail.com a five-year period from 2004 to 2008 and also to determine the drug resistance pattern of Shigella in a tertiary-care hospital in North India.

In this cross-sectional study, patients presenting with acute, bloody diarrhoea, suggestive of shigellosis, during 2004-2008, were included. Stool samples were collected from all patients presenting with symptoms of diarrhoea, blood or mucus in stool, fever, and abdominal pain. In total, 12,983 stool samples were received for culture and routine microscopy during 2004-2008. All the samples were subjected to routine microscopy and were examined for pus cells, trophozoites, and cysts. Only those samples which showed pus cells and/or red blood cells were cultured. The stool specimens from such symptomatic patients were cultured on differential and selective media, including MacConkey agar, Xylose-lysine desoxycholate agar, and deoxycholate citrate agar as primary plates. Loop-full sample was also inoculated into selenite-F broth. The plates were incubated at $37^{\circ} \mathrm{C}$ for 24 hours. Suspected colonies on solid plates, which were Gram-negative upon Gram-staining and oxidase test-negative, were biochemically characterized as per standard procedures. Presumptive Shigella isolates on Triple Sugar Iron agar were serotyped by slide agglutination tests with Shigella polyvalent grouping and monovalent antisera (Denka Seiken Co. Ltd., Tokyo, Japan).

Antimicrobial susceptibility testing and resistance patterns of the Shigella isolates to various antimicrobials were determined by the disc-diffusion technique (10). Every inoculum was prepared by inoculating $5 \mathrm{~mL}$ of Mueller-Hinton broth with five colonies of an 18-hour old pure Shigella culture, followed by incubation in ambient air and at $37^{\circ} \mathrm{C}$ for 16 hours. Turbidity of the broth was equilibrated to match with 0.5 McFarland standards. A sterile cotton swab was dipped into the standardized suspension, drained, and used for inoculating 25 $\mathrm{mL}$ of Mueller-Hinton agar in a $90-\mathrm{mm}$ plate. The inoculating plates were air-dried, and antimicrobial discs, included ampicillin $(10 \mu \mathrm{g})$, chloramphenicol $(30 \mu \mathrm{g})$, co-trimoxazole $(25 \mu \mathrm{g})$, tetracycline $(30 \mu \mathrm{g})$, gentamicin $(10 \mu \mathrm{g})$, nalidixic acid $(30 \mu \mathrm{g})$, 
and ciprofloxacin $(5 \mu \mathrm{g})$. Furazolidone [Oxoid (UK) and Hi-Media (Mumbai, India)] were placed on them. The plates were inverted and incubated in ambient air at $37^{\circ} \mathrm{C}$ for 18 hours. Zone-sizes were measured and compared with those of Escherichia coli ATCC25922 which served as a control strain.

Minimum inhibitory concentrations were determined against ceftriaxone and cefotaxime using broth dilution techniques as per the guidelines of the Clinical Laboratory Standards Institute $(10,11)$. $\mathrm{MIC}_{50}$ and $\mathrm{MIC}_{90}$ were calculated using cumulative frequency table.

In total, 12,983 stool samples were received for culture and routine microscopy during 2004-2008. Of these samples, 2,109 (16.2\%) were from indoor patients while the remaining samples were from outdoor patients. Upon performing routine microscopy, only those samples which showed pus cells and/or red blood cells were cultured. In total, 4,900 such samples were cultured. Shigella species were isolated from 106 such patients-74 (69.8\%) males and 32 (30.1\%) females-during 2004-2008. The age distribution of patients who were positive for Shigella ranged from 23 days to 72 years, with a mean age of 17.66 years. The most commonlyaffected age-group was 1-5 years (39.6\%), followed by the age-group of less than one year (27.3\%). All the four serogroups were isolated with the following distribution: 73 strains of S. flexneri (68.8\%), 26 strains of S. dysenteriae (24.5\%), six strains of S. boydii (5.66\%), and one strain of S. sonnei (0.9\%). Resistance patterns against the applied antimicrobials were as follows: nalidixic acid-94.3\%; ampicillin88.6\%; trimethoprim-sulphamethoxazole-84.9\%, tetracycline-79.2\%; ciprofloxacin-67.9\%; chloramphenicol-54\%; and gentamicin-17.9\% (Table 1).
Twelve distinct resistance patterns were encountered in all the Shigella strains tested (Table 2). One hundred four isolates $(98.11 \%)$ were multiple drugresistant (MDR). Twelve strains were resistant to seven of the eight antimicrobials tested. Sixty-nine strains were resistant to six of the eight antimicrobials.

$\mathrm{MIC}_{90}$ was calculated at $32 \mu \mathrm{g} / \mathrm{mL}$ while $\mathrm{MIC}_{50}$ was found to be $16 \mu \mathrm{g} / \mathrm{mL}$ for both cefotaxime and ceftriaxone. Shigellosis occurs both in epidemic and endemic forms in children and is a major public-health problem in developing countries. This retrospective study demonstrated a high level of antimicrobial resistance pattern in Shigella species isolated from stool samples over a five-year period at a tertiary-care teaching hospital in Delhi, north India.

The majority (67\%) of the Shigella species were isolated from children aged less than five years, which is similar to findings of other studies $(4,8)$. The fact that the majority of Shigella isolates are from the paediatric population is reported in the literature where $70 \%$ of all infections occur in children aged less than 15 years (1).

The frequency of different species of Shigella varies in different countries. The most abundant species of Shigella in the present study was $S$. flexneri which is similar to studies in Zahedan, Iran (2,3), Ghana (4), and western Nepal (5). A multicentre study by Seilden et al. also found $S$. flexneri as the commonest species in all, except Thailand where $S$. sonnei was the common isolate (12). A significant longitudinal transition of species from $S$. flexneri to $S$. sonnei and change in antimicrobial resistance pattern have been observed in Viet Nam by Vinh et al. (13).

\begin{tabular}{|c|c|c|c|c|c|c|c|c|c|c|}
\hline \multirow{3}{*}{$\begin{array}{l}\text { Antimicrobial } \\
\text { drug }\end{array}$} & \multirow{2}{*}{\multicolumn{2}{|c|}{ Resistant isolates }} & \multicolumn{8}{|c|}{ Serogroups } \\
\hline & & & \multicolumn{2}{|c|}{$\begin{array}{l}\text { S. flexneri } \\
(\mathrm{n}=73)\end{array}$} & \multicolumn{2}{|c|}{$\begin{array}{l}\text { S. dysenteriae } \\
(\mathrm{n}=26)\end{array}$} & \multicolumn{2}{|c|}{$\begin{array}{c}\text { S. boydii } \\
(\mathrm{n}=6)\end{array}$} & \multicolumn{2}{|c|}{$\begin{array}{l}\text { S. sonnei } \\
(\mathrm{n}=1)\end{array}$} \\
\hline & No. & $\%$ & No. & $\%$ & No. & $\%$ & No. & $\%$ & No. & $\%$ \\
\hline Ampicillin & 94 & 88.6 & 70 & 95.8 & 20 & 33.3 & 3 & 50 & 1 & 100 \\
\hline Co-trimoxazole & 90 & 84.9 & 69 & 94.5 & 18 & 69.2 & 2 & 33.3 & 1 & 100 \\
\hline Tetracycline & 84 & 79.2 & 58 & 79.4 & 21 & 80.7 & 4 & 66.6 & 1 & 100 \\
\hline Chloramphenicol & 58 & 54 & 46 & 63.3 & 12 & 46.1 & 0 & 00 & 0 & 00 \\
\hline Gentamicin & 19 & 17.9 & 13 & 17.8 & 6 & 23 & 0 & 00 & 0 & 00 \\
\hline Nalidixic acid & 100 & 94.3 & 69 & 94.5 & 26 & 100 & 4 & 66.6 & 1 & 100 \\
\hline Ciprofloxacin & 72 & 67.9 & 44 & 60.2 & 15 & 57.6 & 0 & 00 & 0 & 00 \\
\hline Furazolidone & 4 & 3.77 & 4 & 5.4 & 0 & 00 & 0 & 00 & 0 & 00 \\
\hline Cefotaxime & 19 & 17.9 & 12 & 16.4 & 6 & 23 & 1 & 16 & 0 & 00 \\
\hline Ceftriaxone & 13 & 12.2 & 10 & 13.6 & 3 & 11.5 & 0 & 00 & 0 & 00 \\
\hline
\end{tabular}


However, in our setting, $S$. flexneri continues to predominate.

Over the past decades, Shigella spp. have become progressively more resistant to most widely-used and inexpensive antimicrobials. The last two decades have seen an emergence of multidrug-resistant (MDR) strains of Shigella spp. The World Health Organization currently recommends ciprofloxacin (or other fluoroquinolones) as the drug of choice for therapy of Shigellainfections in both adults and children. In addition, ceftriaxone, pivmecillinam (amdinocillin pivoxil), and azithromycin are also considered alternative drugs suitable for the treatment of Shigella-associated disease (14). However, strains of Shigella spp. that are resistant to cipro-

\begin{tabular}{|c|c|}
\hline Antimicrobials resistance pattern & $\begin{array}{l}\text { No. of } \\
\text { isolates }\end{array}$ \\
\hline \multicolumn{2}{|l|}{$\mathrm{R}$ to 10 antimicrobials } \\
\hline A,T,C,Co,Cf,G,Na,Fx,Ce,Ci & 3 \\
\hline \multicolumn{2}{|l|}{$\mathrm{R}$ to 9 antimicrobials } \\
\hline A,T,C,Co,Cf,G,Na,Ce,Ci & 1 \\
\hline \multicolumn{2}{|l|}{$\mathrm{R}$ to 8 antimicrobials } \\
\hline A,T,C,Co,Cf,G,Na,Fx, & 1 \\
\hline $\mathrm{A}, \mathrm{T}, \mathrm{C}, \mathrm{Co}, \mathrm{Cf}, \mathrm{G}, \mathrm{Na}, \mathrm{Ci}$ & 3 \\
\hline A,T,C,Co,Cf,G,Na,Ce & 2 \\
\hline \multicolumn{2}{|l|}{$\mathrm{R}$ to 7 antimicrobials } \\
\hline A,T,C,Co,Cf,Na,Fx & 2 \\
\hline $\mathrm{A}, \mathrm{T}, \mathrm{C}, \mathrm{Co}, \mathrm{Cf}, \mathrm{Na}, \mathrm{G}$ & 5 \\
\hline $\mathrm{A}, \mathrm{T}, \mathrm{C}, \mathrm{CO}, \mathrm{Ce}, \mathrm{Ci}, \mathrm{G}$ & 8 \\
\hline $\mathrm{A}, \mathrm{T}, \mathrm{Co}, \mathrm{Cf}, \mathrm{Ci}, \mathrm{Na}, \mathrm{Fx}$ & 4 \\
\hline \multicolumn{2}{|l|}{$\mathrm{R}$ to 6 antimicrobials } \\
\hline $\mathrm{A}, \mathrm{C}, \mathrm{C} 0, \mathrm{~T}, \mathrm{Cf}, \mathrm{Na}$ & 50 \\
\hline $\mathrm{A}, \mathrm{C}, \mathrm{Co}, \mathrm{T}, \mathrm{Cf}, \mathrm{Ce}$ & 2 \\
\hline $\mathrm{A}, \mathrm{C}, \mathrm{CO}, \mathrm{T}, \mathrm{Cf}, \mathrm{Ci}$ & 2 \\
\hline $\mathrm{A}, \mathrm{C}, \mathrm{Co}, \mathrm{Cf}, \mathrm{G}, \mathrm{T}$ & 7 \\
\hline \multicolumn{2}{|l|}{$\mathrm{R}$ to 5 antimicrobials } \\
\hline $\mathrm{T}, \mathrm{C}, \mathrm{Co}, \mathrm{Cf}, \mathrm{Na}$ & 6 \\
\hline A,T,C,Co,Cf & 4 \\
\hline $\mathrm{A}, \mathrm{T}, \mathrm{C}, \mathrm{Cf}, \mathrm{Na}$ & 1 \\
\hline \multicolumn{2}{|l|}{$\mathrm{R}$ to 4 antimicrobials } \\
\hline $\mathrm{A}, \mathrm{C}, \mathrm{Cf}, \mathrm{T}$ & 1 \\
\hline \multicolumn{2}{|l|}{$\mathrm{R}$ to 3 antimicrobials } \\
\hline $\mathrm{C}, \mathrm{Co}, \mathrm{Cf}$ & 1 \\
\hline A,C,Co & 1 \\
\hline Total & 104 \\
\hline
\end{tabular}

$\mathrm{A}=$ Ampicillin; $\mathrm{C}=$ Chloramphenicol; $\mathrm{Ce}=$ Ceftriaxone; $\mathrm{C}=$ =Ciprofloxacin; $\mathrm{Ci}=$ Cefotaxime; $\mathrm{Co}=$ Co-trimoxazole; $\mathrm{Fu}=$ Furazolidone; $\mathrm{G}=$ Gentamicin; $\mathrm{Na}=$ Nalidixic acid; $\mathrm{T}=$ Tetracycline floxacin have been described in Asia $(13,15)$. Also, strains of Shigella spp. producing extended-spectrum $\beta$-lactamases (ESBL) conferring resistance to third-generation cephalosporins have also been reported (16).

S. flexneri, the commonest isolate in our study, showed a high degree of resistance to most commonly-used drugs, such as nalidixic acid (94.3\%), ampicillin $(95.8 \%)$, co-trimoxazole $(94.5 \%)$, tetracycline (79.4\%), ciprofloxacin (60.2\%), and chloramphenicol (63.3\%).

In this study, multiple drug resistance to as many as seven antimicrobials was observed. Similar findings were reported in other studies from other geographic areas, such as from Ethiopia and recently from Viet Nam $(1,13)$.

Most S. flexneri isolates in our study were susceptible to gentamicin and furazolidone which support the findings of the study conducted by Taneja et al. (17). However, the relative increase in the resistance to ciprofloxacin (67.2\%) in the study by Neogi in all Shigella spp., compared to other studies $(2,3,5-$ 7,16 ), indicates that there is a great need to reduce the indiscriminate use of potent antimicrobials, such as ciprofloxacin (15).

Some degree of regulation of antimicrobial use is necessary as most antimicrobials are available overthe-counter and can be bought without any prescription. There is a need to educate both general public and health practitioners that most cases of diarrhoea do not require antimicrobials. Periodic monitoring of drug resistance in enteropathogens should be carried out in different geographic areas so that an appropriate agent can be chosen for empiric therapy. Currently, there are effective optional therapies, and ceftriaxone is used as an alternative when the patient does not respond to treatment with fluoroquinolone. A marked increase in resistance to nalidixic acid and newer fluoroquinolones, combined with ESBL-mediated third-generation cephalosporin resistance, would leave limited treatment options for those with life-threatening bacterial infections.

\section{REFERENCES}

1. Yismaw G, Negeri C, Kassu A. A five-year antimicrobial resistance pattern observed in Shigella species isolated from stool samples in Gondar University Hospital, northwest Ethiopia. Ethiop J Health Dev 2006;20:194-8.

2. Qureishi MI, Borji A, Bokaeian M, Roudbari M, Shahraki S, Niazi A et al. Antimicrobial resistance of Shig- 
ella spp. isolated from diarrheal patients in Zahedan. Acta Med Iranica 2008;46:413-6.

3. Mamatha B, Pusapati BR, Rituparna C. Changing patterns of antimicrobial susceptibility of Shigella serotypes isolated from children with acute diarrhoea in Manipal South India-a 5-year study. Southeast Asian J Trop Med Public Health 2007;38:863-6.

4. Opintan J, Newman MJ. Distribution of serogroups and serotypes of multiple drug resistant Shigella isolates. Ghana Med J 2007;41:8-29.

5. Savadkoohi R, Mousa A. Prevalence of Shigella species and their antimicrobial resistance patterns at Amirkola Children's Hospital, North Iran. Iran J Pediatr 2007;17:118-22.

6. Bhattacharya S, Khanal B, Bhattarai NR, Das ML. Prevalence of Shigella species and their antimicrobial resistance patterns in eastern Nepal. J Health Popul Nutr 2005;23:339-42.

7. Hosseini MJ, Ranjbar R, Ghasemi H, Jalalian HR. The prevalence and antimicrobial resistance of Shigella sp. recovered from patients admitted to Bouali Hospital, Tehran, Iran during 1999-2001. Pak J Biol Sci 2007;10: 2778-80.

8. Shrestha CD, Malla S, Maharjan L. Multi drug resistant Shigella species in Nepal, a retrospective study conducted at National Public Health Laboratory (NPHL), 1999 to 2002. J Nepal Health Res Counc 2002;4:51-5.

9. Wilson G, Easow JM, Mukhopadhyay C, Shivananda PG. Isolation \& antimicrobial susceptibility of Shigella from patients with acute gastroenteritis in western Nepal. Ind J Med Res 2006;123:145-50.

10. Clinical and Laboratory Standards Institute. Perform- ance standards for antimicrobial susceptibility testing, seventeenth informational supplement. Wayne, PA: Clinical and Laboratory Standards Institute, 2007;27:32-6.

11. Andrews JM. Determination of minimum inhibitory concentrations. J Antimicrob Chemother 2001;43:516.

12. von Seidlein L, Kim DR, Ali M, Lee H, Wang X, Thiem VD et al. A multicentre study of Shigella diarrhoea in six Asian countries: disease burden, clinical manifestations, and microbiology. PLoS Med 2006;3:353.

13. Vinh H, Nhu NT, Nga TV, Duy PT, Campbell JI, Hoang $\mathrm{NV}$ et al. A changing picture of shigellosis in southern Vietnam: shifting species dominance, antimicrobial susceptibility and clinical presentation. BMC Infect Dis 2009;9:204.

14. World Health Organization. Guidelines for the control of shigellosis, including epidemics due to Shigella dysenteriae type 1 . Geneva: World Health Organization, 2005:11-3.

15. Niyogi SK. Shigellosis. J Microbiol 2005;43:133-43.

16. Fortineau N, Naas T, Gaillot O, Nordmann P. SHV-type extended-spectrum $\beta$-lactamase in a Shigella flexneri clinical isolate. J Antimicrob Chemother 2001;47:685-8.

17. Taneja N, Mohan B, Khurana S, Sharma M. Antimicrobial resistance in selected bacterial enteropathogens in north India. Indian J Med Res 2004;120:3943.

\section{Urvashi, Sonal Saxena, and Renu Dutta}

Department of Microbiology, Lady Hardinge Medical College and Associated Hospitals, New Delhi, India 